Chirurg 2017 $\cdot 88: 710$

DOI 10.1007/s00104-017-0478-0

Online publiziert: 20. Juli 2017

(c) Springer Medizin Verlag GmbH 2017

CrossMark
W. Schröder · C. Bruns

Klinik für Allgemein-, Viszeral- und Tumorchirurgie, Universitätsklinik Köln, Köln, Deutschland

\section{Das Siegelringzellkarzinom des Magens}

\section{Neue Aspekte zur Langzeitprognose}

\section{Originalpublikation}

Chon HJ, Hyung WJ, Kim C et al (2017)

Differential prognostic implications of gastric signet ring cell carcinoma. Stage adjusted analysis from a single high-volume center in Asia. Ann Surg 265:946-953

Hintergrund und Fragestellung. Das Siegelringzellkarzinom (SRC) des Magens ist eine histopathologische Variante des tubulären Adenokarzinom (AC) des Magens. Der Einfluss des SCR auf die Langzeitprognose und die damit verbundenen therapeutischen Strategien sind Gegenstand aktueller Diskussionen, ohne dass hier wegen der unzureichenden Datenlage zwischen Frühstadien und lokal fortgeschrittenen Tumoren differenziert wird.

Methoden. In diese retrospektive Analyse eines einzelnen „High-volume“-Zentrums in Südkorea wurden von Januar 2001 bis Dezember 20107667 Patienten mit histologisch gesichertem Magenkarzinom eingeschlossen, die nicht vorbehandelt, sondern primär mittels Gastrektomie mit D2-Lymphadenektomie standardisiert operiert wurden (R0-Resektion). Basierend auf der histopathologischen WHO-Klassifikation des Primärtumors wurden 3 Gruppen verglichen: 1646 Patienten mit SCR, 3403 Patienten mit einem gut bis mäßig differenzierten tubulären AC und 2618 Patienten mit einem schlecht differenzierten AC. Das mediane Follow-up aller Patienten lag bei 63,9 Monaten. Die Patientendaten wurden insbesondere hinsichtlich möglicher Prognosefaktoren des SRC aufgearbeitet.
Ergebnisse. Patienten mit SRC waren mit einem Durchschnittalter von 52 Jahren bei Diagnosestellung signifikant jünger als Patienten mit tubulärem Adenokarzinom (gut/mäßig differenziertes AC: 61 Jahre, schlecht differenziertes AC: 56 Jahre; $p<0,001)$. Beim SCR war der Anteil von Frauen mit 50,6 \% deutlich höher als beim tubulärem AC (gut/mäßig differenziertes AC: $22,8 \%$, schlecht differenziertes AC: 38,2\%; $p<0,001) .61,9 \%$ der Patienten mit SRC wurden im Frühstadium (IA nach der AJCC [American Joint Committee on Cancer], 7. Ausgabe) diagnostiziert. Der überwiegende Anteil der SCR (49,8 \%) war im unteren Drittel des Magens lokalisiert, 96,4\% des SRC wurden nach Lauren als diffuser Typ klassifiziert. Eines der wesentlichen Ergebnisse dieser Arbeit ist, dass Patienten mit Frühstadium eines SCR eine signifikant bessere Langzeitprognose aufwiesen als Patienten mit tubulärem AC unabhängig vom Differenzierungsgrad des Primärtumors (10-Jahres-Überleben: $95 \%$ vs. $85 \%, p<0,001)$. Dieser Effekt ließ sich aber für lokal fortgeschrittene AC nicht mehr nachweisen (10-Jahres-Überleben: $53 \%$ vs. $54 \%, p=0,049$ ). Bei getrennter Betrachtung der lokal fortgeschrittenen tubulären AC zeigte sich jedoch, dass gut bis mäßig differenzierte $\mathrm{AC}$ ein besseres Langzeitüberleben hatten als $\mathrm{Pa}$ tienten mit SRC oder schlecht differenziertem AC (10-Jahres-Überleben: SRC $53 \%$, schlecht differenziertes AC 52\%, gut bis mäßig differenziertes AC $58 \%$, $p<0,001)$.

Fazit. Die vorliegende retrospektive Analyse ist das größte Patientenkollektiv mit SCR des Magens, welches in einem der weltweit größten Zentren für onkologische Magenchirurgie operiert wurde. Die Daten zeigen überraschenderweise, dass das rezidivfreie und Langzeitüberleben von Patienten mit SCR im Frühstadium besser ist als von Patienten mit dem klassischen gut bis mäßig differenzierten AC. Diese gute Prognose ändert sich mit Progress zum lokal fortgeschrittenen Karzinom. Trotz einer niedrigen Prävalenz in den westlichen Ländern ist nach diesen Daten die generelle Empfehlung der aktuellen S3-Leitlinie „Magenkarzinom“ zur Gastrektomie bei SCR in den frühesten Stadien, die immer als diffuser Typ nach Lauren imponieren, zu überdenken.

\section{Korrespondenzadresse}

Prof. Dr. W. Schröder, FACS, FEBS

Klinik für Allgemein-, Viszeral- und

Tumorchirurgie, Universitätsklinik Köln Kerpener Str. 62, 50937 Köln, Deutschland wolfgang.schroeder@uni-koeln.de

Interessenkonflikt. W. Schröder und C. Bruns geben an, dass kein Interessenkonflikt besteht. 